\title{
PERFIL EPIDEMIOLÓGICO DA TOXOPLASMOSE CONGÊNITA NO ESTADO DE SANTA CATARINA
}

\section{Epidemiological profile of congenital toxoplasmosis in the state of Santa Catarina}

https://doi.org/10.18593/eba.28575

Recebido em 30 de agosto de 2021 | Aceito em 06 de novembro de 2021

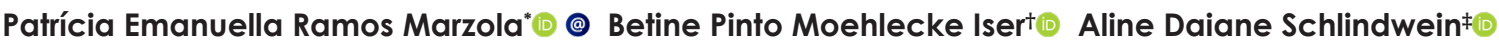

Mestra em Ciências da Saúde pela Universidade do Sul de Santa Catarina; Universidade do Sul de Santa Catarina, Programa de PósGraduação em Ciências da Saúde.

Doutora em Epidemiologia pela Universidade Federal do Rio Grande do Sul.

‡ Doutora em Biotecnologia e Biociências pela Universidade Federal de Santa Catarina.

Resumo: Introdução - A toxoplasmose é uma doença infecciosa causada pelo Toxoplasma gondii, que pode ser transmitida ao feto caso a mulher seja infectada durante o período gestacional. Muitas crianças são assintomáticas ao nascimento, contudo, podem apresentar sequelas graves ao longo da vida. Objetivo - Descrever as informações epidemiológicas da toxoplasmose congênita no estado de Santa Catarina. Metodologia - Trata-se de um estudo ecológico, com base em dados secundários oficiais. Foram avalias informações referentes ao período de 2010 a 2020. Resultados - Observou-se uma alta incidência de toxoplasmose congênita, especialmente nas regiões da Foz do Itajaí, Nordeste e Planalto Norte, e da Grande Florianópolis, com valores de 8,79, 6,04 e 5,02 casos por 10.000 nascidos vivos, respectivamente. Salienta-se a presença de alta incidência entre indígenas, com 3,3 casos a cada 100 nascidos vivos. Conclusões - Apesar do progresso em relação à notificação e monitoramento da toxoplasmose gestacional e congênita no estado catarinense, foram identificadas falhas no processo de notificação do recém-nascido, consequentemente, com perda de acompanhamento e tratamento. Em função da alta incidência da toxoplasmose gestacional e congênita, recomenda-se a introdução de medidas educacionais para orientação dessa população. Destaca-se a necessidade de atenção à população indígena.

Palavras-chave: Epidemiologia. Notificação de doenças infecciosas. Transmissão vertical de doenças infecciosas.

Abstract: Introduction-Toxoplasmosis is an infectious disease that can be transmitted to the fetus if the woman is infected during pregnancy. Many children are asymptomatic at birth; however, several can have severe sequelae throughout their lives. Objective - To describe the epidemiological information of congenital toxoplasmosis in the state of Santa Catarina. Methodology - This is an ecological study, based on official secondary data. There was evaluated information for the period 2010 to 2020. Results - There was a high incidence of congenital toxoplasmosis, especially in the regions of Foz do Itajaí, Northeast and North Plateau, and Greater Florianópolis, with incidences of 8.79, 6, 04 and 5.02 cases per 10,000 live births, respectively. The presence of a high incidence among indigenous people is highlighted, with 3.3 cases per 100 live births. Conclusions - Despite the progress regarding notification and monitoring of gestational and congenital toxoplasmosis in the state of Santa Catarina, failures were identified in the newborn notification process, consequently, with loss of follow-up and treatment. Due to the high incidence of gestational and congenital toxoplasmosis, the introduction of educational measures to guide this population is recommended. The need for attention to the indigenous population is highlight.

Keywords: Epidemiology. Infectious disease notifications. Vertical transmission of infectious diseases.

@ Autor correspondente: Mestra em Ciências da Saúde pela Universidade do Sul de Santa Catarina; Universidade do Sul de Santa Catarina, Programa de Pós-Graduação em Ciências da Saúde, Avenida Pedra Branca, 744, Palhoça, CEP 88137-247; ORCID: https://orcid.org/00000001-8152-5674; patriciarmarzola@gmail.com 


\section{INTRODUÇÃO}

A toxoplasmose é uma doença parasitária em que pode ocorre a transmissão materno-fetal do Toxoplasma gondii caso a infecção ocorra durante o período gestacional, ocasionado a toxoplasmose congênita. Em cerca de 50\% dos casos a infecção congênita é assintomática ${ }^{1}$, mas os sintomas podem surgir ao longo dos primeiros anos de vida ou até mesmo na adolescência. As principais manifestações clínicas observadas são as lesões oftalmológicas (retinocoroidite), além de malformações congênitas, lesões neurológicas, atraso no desenvolvimento, prematuridade, abortamento ou a morte ${ }^{1,2}$.

O diagnóstico da toxoplasmose congênita é complexo, e deve considerar o binômio maternofetal. A presença da soroconversão materna, em qualquer trimestre gestacional, confirmada ou com alta suspeição, indica a necessidade de investigação da criança logo após o nascimento. Para diagnóstico da criança, devem ser solicitadas as sorologias IgM e/ou IgA e IgG nos casos em que há suspeita de transmissão vertical, além de outros exames de imagem, fundo de olho e líquor ${ }^{2}$. Contudo, existem diversos fatores que contribuem para resultados falso-positivos e falso-negativos ao nascimento, sendo recomendado que a criança seja encaminhada a centros especializados para confirmação diagnóstica e indicação terapêutica. De forma geral, os critérios para diagnóstico envolvem a presença da imunoglobulina $\operatorname{IgM} / \operatorname{IgA}$ após 10 dias de vida; a persistência da positividade da sorologia IgG ao longo do primeiro ano de vida; ou a associação das manifestações clínicas clássicas com a positividade da IgG, nos casos em que as gestantes foram confirmadas para a infecção, sendo descartadas outras doenças ${ }^{1,2}$.
Tendo em vista a dificuldade diagnóstica da toxoplasmose congênita e considerando os benefícios da triagem materna e fetal para introdução do tratamento precoce em recémnascidos, este trabalho tem como objetivo estudar a epidemiologia da toxoplasmose congênita no estado de Santa Catarina, no período de 2010 a 2020 .

\section{MÉTODOS}

Trata-se de um estudo ecológico, com análisededadossecundários. Asinformações foram obtidas a partir do banco de dados disponibilizado no Sistema Estadual de Informações e Agravos de Notificação ${ }^{3}$ e no banco de dados de Nascidos Vivos $^{4}$, ambos da Diretoria de Investigação em Saúde de Santa Catarina. Foram pesquisadas as informações referentes ao período de 2010 a 2020. A seleção do período foi realizada com base na disponibilidade das informações.

Foram investigadas as seguintes informações referentesaoestadodeSanta Catarina: o número de nascidos vivos, classificados por ano de nascimento, macrorregião de ocorrência, sexo, cor/raça e zona de habitação; número de gestantes com toxoplasmose gestacional notificadas e confirmadas, classificadas por ano de notificação e macrorregião de ocorrência; e o número de casos de toxoplasmose congênita notificados e confirmados, classificados de acordo com o ano e macrorregião de ocorrência, sexo, cor/raça, zona de habitação e evolução.

Salienta-se que os casos de toxoplasmose gestacional econgênitasãoinicialmentereportados ao banco de dados como sendo "notificados", e são alterados para "confirmados" ou "descartados" de acordo com a evolução da investigação. Assim sendo, nesta pesquisa, serão considerados 
"notificados" todos os casos suspeitos e reportados ao sistema oficial de notificação, e "confirmados", todos os casos que foram reportados e que apresentam confirmação diagnóstica.

Para o cálculo da taxa de incidência das informações referentes às macrorregiões e do estado de Santa Catarina, utilizou-se como base a população residente em todo estado ou nas respectivas macrorregiões. As informações do banco de dados foram consideradas como "casos novos", tendo em vista que o diagnóstico da toxoplasmose congênita acontece nos primeiros meses de vida. Assim sendo, os valores de incidência coincidem com os valores de prevalência da doença ${ }^{5}$.

A taxa de transmissão vertical foi calculada com base no número de casos confirmados de toxoplasmose gestacional e o número de casos de toxoplasmose congênita confirmados.

Oaumentopercentualdecasos foi estimado considerando o valor final subtraído do valor inicial, divididos pelo valor inicial multiplicado pelo fator 100. Além disso, os dados foram apresentados como média, frequência absoluta ou relativa e foi realizado o teste do chi-quadrado, quando necessário. As análises estatísticas foram realizadas no software Microsoft Excel. Os dados foram considerados estatisticamente significativos quando o valor de "p" foi menor do que o,05.

Os bancos de dados utilizados pertencem aos sistemas oficiais de informação de saúde, de domínio público e sem identificação individual, de modo que não há possibilidade de violação ética.

\section{RESULTADOS}

\subsection{TOXOPLASMOSE GESTACIONAL}

Entre o ano de 2010 e 2020, foram notificados 1.194 casos de toxoplasmose gestacional no estado de Santa Catarina, sendo que a maioria $(\mathrm{n}=1.052 ; 96,16 \%)$ foi confirmada para a patologia (tabela 1). A média de casos registrados entre 2011 e 2015 foi de 2,5 por ano, enquanto a partir de 2016 , foi de 236,8 ao ano. Entre o ano de 2010 e 2020 , observou-se um aumento percentual de $18.850 \%$ das notificações. Quando considerado a partir de 2016, o aumento percentual foi de cerca de $1.700 \%$.

Tabela 1 - Frequências absoluta e relativa de casos notificados de toxoplasmose gestacional, classificadas por macrorregião, no período de 2010 a 2020.

\begin{tabular}{lrr}
\hline \multicolumn{1}{c}{ Macrorregião } & $\begin{array}{c}\text { Frequência } \\
\text { absoluta } \\
\text { por região }\end{array}$ & $\begin{array}{c}\text { Frequência } \\
\text { relativa } \\
\text { por região (\%) }\end{array}$ \\
\hline Grande Oeste & 98 & 8,21 \\
Meio Oeste e Serra & 216 & $\mathbf{1 8 , 0 9}$ \\
Vale do Itajaí & 223 & $\mathbf{1 8 , 6 8}$ \\
Foz do Rio Itajaí & 97 & 8,12 \\
Grande & 137 & $\mathbf{1 1 , 4 7}$ \\
Florianópolis & 77 & 6,45 \\
Sul & 346 & $\mathbf{2 8 , 9 8}$ \\
Nordeste e Planalto & $\mathbf{1 1 9 4}$ & $\mathbf{1 0 0}$ \\
Norte & & \\
\hline Total & &
\end{tabular}

Na Figura 1, é possível visualizar o aumento do número de casos de toxoplasmose gestacional confirmados e o número de casos investigados de toxoplasmose congênita ao longo do período investigado. Salienta-se, contudo, que o número de casos investigados são aproximadamente 6o\% menores do que o número de gestantes com toxoplasmose confirmada. 


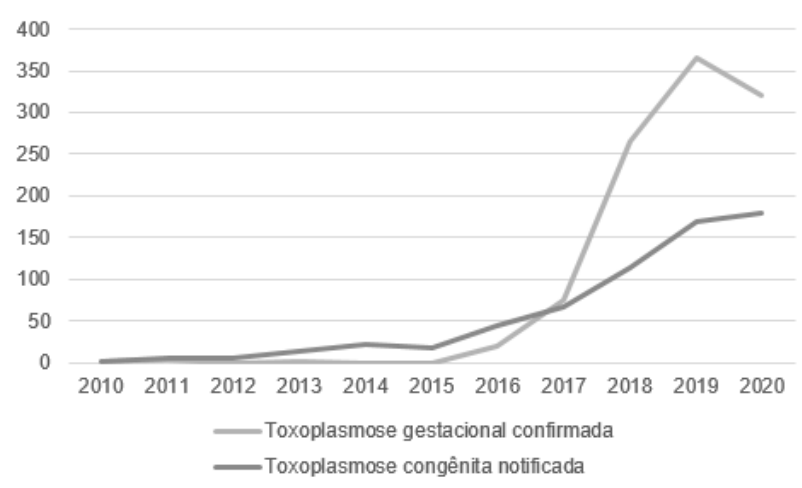

Figura 1 - Distribuição de casos de toxoplasmose gestacional e congênita no estado de Santa Catarina, entre 2010 e 2020 .

\subsection{TOXOPLASMOSE CONGÊNITA}

No período avaliado, foram notificados 636 casos com suspeita de toxoplasmose congênita, sendo que destes, 450 (70\%) foram notificados como casos confirmados entre os recém-nascidos. A taxa de transmissão vertical, nesta amostra, foi de $42,7 \%$.

Dos casos confirmados de toxoplasmose congênita, 228 eram de crianças do sexo masculino. Não foi observada diferença estatisticamente significativa entre os sexos das crianças ea presença da toxoplasmose congênita $(\mathrm{p}=0,2980)$.

Dos 450 casos de toxoplasmose congênita, $380 \quad(84,44 \%)$ apresentavam informações relacionadas ao ambiente em que vivem, sendo que $90,79 \% \quad(n=345)$ eram de áreas urbanas; $7,89 \%(\mathrm{n}=30)$ de área rural e $1,32 \%(\mathrm{n}=5)$ de área periurbana.

Na análise da cor de pele, não foi identificada diferença estatisticamente significativa entre brancos e pretos, amarelos ou pardas. Contudo, houve uma diferença significativa quando comparadas com as crianças indígenas $(\mathrm{p}=0,0044)$. No período em questão, foram registrados 90 nascimentos de crianças indígenas, sendo que três apresentaram toxoplasmose congênita confirmada, o que representa uma incidência de 3,3 casos a cada 100 nascidos vivos. Todos os casos relacionados a indígenas ocorreram na região do Grande Oeste.

Foi registrado um óbito entre os casos confirmados. Não há informações sobre abortamento, natimortos e a morbidade da doença.

As regiões com maior número absoluto de notificações foram: Norte e Planalto Norte $(n=148)$, sendo que $85 \%$ destes foram confirmados; Vale do Itajaí ( $n=116$ ), sendo cerca de $45 \%$ confirmados; Foz do Itajaí ( $n=116)$, sendo que $81 \%$ destes foram confirmados; e Grande Florianópolis $(n=112)$ em que $75 \%$ foram confirmados.

A incidência da toxoplasmose congênita no estado de Santa Catarina, no período de 2011 a 2020, foi de 4,37 casos a cada 10.000 nascidos vivos (Tabela 2). As regiões com incidências mais altas foram Foz do Itajaí, Nordeste e Planalto Norte e Grande Florianópolis. Já as regiões que foram significativamente menores que a média estadual foram o Meio Oeste e Serra e Sul. 
Tabela 2 - Número de casos de toxoplasmose congênita notificados e confirmados e a incidência da doença no estado de Santa Catarina, no período de 2011 a 2020, classificados por macrorregião de saúde.

\begin{tabular}{llllll}
\hline $\begin{array}{c}\text { Casos de Toxoplasmose } \\
\text { Congênita }\end{array}$ & Nascidos vivos & Notificados & Confirmados & $\begin{array}{c}\text { Incidência (por } \\
\text { 10.00o habitantes) }\end{array}$ & p-valor \\
\hline Nordeste e Planalto Norte & 208.460 & 148 & 126 & 6,04 & 0,0013 \\
Vale do Itajaí & 148.088 & 116 & 53 & 3,58 & 0,1662 \\
Foz do Itajaí & 108.082 & 116 & 95 & 8,79 & $<0.0001$ \\
Grande Florianópolis & 167.273 & 112 & 84 & 5,02 & 0,2456 \\
Grande Oeste & 117.734 & 49 & 43 & 3,66 & 0,2576 \\
Meio Oeste e Serra & 140.037 & 35 & 20 & 1,43 & $<0.0001$ \\
Sul & 138.959 & 60 & 29 & 2,09 & $<0.0001$ \\
\hline Total & 1.028 .633 & 636 & 450 & 4,37 & \\
\hline Fonte: DIVE3. & & & & &
\end{tabular}

\section{DISCUSSÃO}

Esta análise de dados secundários ilustrou algumas particularidades dos casos de toxoplasmose gestacional e congênita notificados ao sistema oficial de saúde do estado de Santa Catarina, no período de 2010 a 2020. Foram confirmados 1.052 casos de toxoplasmose gestacional no período, com aumento do número de notificações a partir de 2016, conforme esperado pela inserção da doença na lista de notificação². Uma série temporal realizada na França, entre os anos de 1995 e 2010, relatou que a prevalência da infecção de toxoplasmose aguda em gestantes diminuiu de $54,3 \%$ para $36,7 \%$ nos respectivos $\operatorname{anos}^{6}$. Não foi possível realizar o cálculo de prevalência de toxoplasmose aguda gestacional na amostra em função da falta de informações nos bancos de dados oficiais.

Apesar do aumento de notificações, de acordo com os critérios diagnósticos propostos pelo Ministério da $S_{a u ́ d e}{ }^{2}$, esperava-se que, no mínimo, os recém-nascidos de todas as gestantes que tiveram confirmação de toxoplasmose gestacional tivessem acompanhamento para investigação da possibilidade de transmissão vertical. Contudo, constatou-se que menos da metade dos casos foi investigado e notificado. $\mathrm{O}$ fato pode estar relacionado com problemas do sistema de notificação ou de encaminhamento da doença, resultando, consequentemente, no atraso ou ausência do tratamento em lactentes. $\mathrm{O}$ Ministério da Saúde ${ }^{7}$ recomenda a instituição do tratamento logo após os primeiros meses de vida de modo a reduzir as sequelas da doença.

Em Santa Catarina, foram confirmados 450 casos de toxoplasmose gestacional no período estudado. A taxa de transmissão identificada $(42,77 \%)$ possivelmente é subestimada, tendo em vista que a maioria das crianças nascidas de gestantes confirmadas para toxoplasmose não foram investigadas. De acordo com a literatura ${ }^{1}$, a taxa de transmissão varia de acordo com o período gestacional em que ocorre a soroconversão materna, de $15 \%$ no 1 - trimestre gestacional, até $70 \%$ no $3^{\circ}$ trimestre. Corroborando com os dados encontrados nesta pesquisa, Martin ${ }^{8}$ cita a taxa de transmissão geral, independente do trimestre de soroconversão materna, de 40\%.

Dos casos confirmados de toxoplasmose congênita, 228 eram de crianças do sexo masculino. 
Não foi observada diferença estatisticamente significativa entre os sexos das crianças e a presença da toxoplasmose congênita $(\mathrm{p}=0,9225)$. Essa informação corrobora com o relatado por Jones et al. 9 Em um estudo de caso controle, que avaliou a população estadunidense em geral, os autores não identificaram diferença entre o risco de infecção e o sexo 9 .

A predominância de casos de toxoplasmose congênita na região urbana vai de encontro aos dados encontrados por Antinarelli et al. ${ }^{10}$, que identificaram maior concentração de casos na região rural e periurbana em uma análise espacial realizada no estado de Minas Gerais. Como justificativa às diferenças entre os estudos, sugere-se que a população da zona rural tenha menor acesso aos serviços de saúde e, desta forma, não sejam identificados em estudos com o delineamento como desta pesquisa.

$\mathrm{Na}$ análise de raça/cor entre as crianças com toxoplasmose congênita confirmada, destacou-se a alta incidência entre indígenas. Não foram identificados, na literatura, fatores de risco relacionados à raça ou etnia. Contudo, Garnelo et al. ${ }^{11}$ descrevem que a assistência pré-natal entre indígenas no Brasil é muito precária. Segundo os autores, cerca de $86 \%$ das mulheres indígenas realizaram algum tipo de tratamento pré-natal, entretanto, de forma insatisfatória. Em relação a realização das sorologias anti-Toxoplasma, apenas 32,6\% realizaram-nas, o que possivelmente está associado a uma altíssima subnotificação e incidência maior do que as encontradas nesta pesquisa.

A incidência de toxoplasmose congênita foi de aproximadamente quatro casos a cada 10.000 nascidos vivos. Este dado é superior ao relatado na literatura: 0,5 a 0,82 casos por 10.000 nascidos vivos $^{12}$ nos Estados Unidos; na Áustria, 8,5/10.00o nascidos vivos ${ }^{13}$; na Dinamarca, de 1,9 casos por 10.000 nascidos vivos ${ }^{14}$.

Assim como ocorre em outras doenças, o diagnóstico da toxoplasmose congênita é uma arte: envolve pelo menos dois especialistas, além de uma série de exames laboratoriais, de imagem ou molecular de dois indivíduos, a mãe e filho, associados a manifestações clínicas. Mesmo entre especialistas, o diagnóstico é considerado difícil ${ }^{15}$, especialmente pela ausência de diversas informações ao longo da gestação e dos primeiros exames no recém-nascido e por particularidades da doença.

No Brasil, a partir do ano de $2018^{2}$, a toxoplasmose gestacional e congênita tornaramse doenças de notificação compulsória, o que beneficia esse processo diagnóstico. Nota-se na presente pesquisa, um aumento do número de notificações da toxoplasmose gestacional e congênita a partir dessa exigência. Todavia, muitas vezes, não existe uma boa comunicação entre os serviços de saúde, públicos ou privados, de forma que muitas informações acabam sendo perdidas durante esse processo. Isso se agrava na toxoplasmose congênita, pois seu diagnóstico depende da avaliação do binômio mãe/filho.

Nesta pesquisa, esperava-se um número maior de casos de crianças avaliadas para toxoplasmose congênita em função do número maior de casos de toxoplasmose gestacional. De acordo com o Ministério da Saúde², toda criança nascida de mãe confirmada ou com provável infecção por toxoplasmose gestacional deve ser investigada quanto à presença de toxoplasmose congênita. A ausência de investigação pode estar relacionada com a subnotificação da doença e 
ausência de tratamento e estimulação precoce de crianças infectadas.

A partir do ano de 2021, o Ministério da Saúde $^{16}$ incluiu a sorologia IgM anti-Toxoplasma no teste do pezinho, o que proporciona uma maior chance de diagnóstico em crianças a despeito dos testes maternos, tendo em vista que é realizado de forma universal. Contudo, é preciso que os profissionais de saúde atentem ao fato de que a sorologia IgM apresenta altos índices de falsonegativo. Desta forma, em casos de crianças cujas mães são suspeitas ou prováveis, deve-se continuar a investigação, mesmo no caso de sorologias IgM negativas.

No estado de Santa Catarina, foi instituída, a partir da Nota Técnica Conjunta $n^{\circ}$ oo8/2019 DAPS/DIAF/DIVE/LACEN ${ }^{17}$, a obrigatoriedade da ficha de notificação compulsória para que a criança receba o tratamento para toxoplasmose congénita no estado, que é centralizado pelo Sistema Único de Saúde. A medida favorece o aumento das notificações dos casos. Todavia, também pode atuar como um empecilho ao acesso rápido ao medicamento, principalmente em uma doença que exige medicação precoce a fim de evitar sequelas neurológicas e oftalmológicas.
Como limitações deste estudo, cita-se o uso de fontes de dados secundários, além do curto período de análise. Existe uma grande chance de subnotificação de dados, de forma que a incidência pode ser muito maior do que a encontrada.

\section{CONCLUSÃO}

Observa-se um avanço nas tentativas de notificação e monitoramento de casos de toxoplasmose gestacional e congênita através de medidas nacionais e regionais. Contudo, relata-se um descompasso entre a notificação materna e do recém-nascido, evidenciando falhas no sistema de encaminhamento das crianças. Recomenda-se a orientação das equipes de saúde na investigação e correta interpretação dos resultados das sorologias maternas e da criança. O estado de Santa Catarina apresentou altas incidências da infecção, especialmente nas macrorregiões da Foz do Rio Itajaí, Nordeste e Planalto Norte e Grande Florianópolis. Destaca-se a necessidade de maiores ações preventivas nessas regiões, assim como investigações acerca das diferenças entre as incidências nas diferentes regiões estaduais. Da mesma forma, recomenda-se que sejam realizadas medidas de orientação e prevenção à toxoplasmose gestacional, especialmente entre populações indígenas.

\section{REFERÊNCIAS}

1. Kota AS, Shabbir N. Congenital Toxoplasmosis. 2021 Jun 29. In: StatPearls. Treasure Island (FL): StatPearls Publishing; 2021. In: PMID 31424812.

2. Brasil, Ministério da Saúde. Protocolo de notificação e investigação: Toxoplasmose gestacional e congênita. Brasília: Ministério da Saúde; 2018. [Internet] [acesso em 2021 Fev 1]. Disponível em: http://bvsms.saude.gov.br/bvs/publicacoes/protocolo_notificacao_. 
3. Diretoria de Vigilância Epidemiológica (DIVE). Notificação Individual. [Internet] [acesso em 2021 Fev 1]. Disponível em: http://200.19.223.105/cgi-bin/dh?sinan/def/notindiv.def.

4. Diretoria de Vigilância Epidemiológica (DIVE). Nascidos Vivos. [Internet] [acesso em 2021 Fev 1]. Disponível em: http://200.19.223.105/cgi-bin/dh?sinasc/def/sinasc.def.

5. Fletcher R. Epidemiologia clínica: elementos essenciais. 5a ed. Porto Alegre: Artmed; 2014.

6. Tourdjman M, Tchéandjieu C, De Valk H, Goulet V LSY. Séroprévalence de la toxoplasmose chez les femmes enceintes en France : évolution entre 1995 et 2010 et facteurs associés. Bull Epidemiol Hebd. 2015;264-72. [Internet] [acesso em 2021 Fev 1]. DOI: 10.3166/bspe-2019-0078.

7. Brasil. Ministério da Saúde. Nota Técnica no 14/2020-COSMU/CGCIVI/DAPES/SAPS/MS; 2020. [Internet]. [acesso em $2021 \mathrm{Fev}$ 1]. Disponível em: https://antigo.saude.gov.br/images/pdf/2020/ July/29/SEI-MS---0014746811---Nota-T--cnica--1-.pdf.

8. Martin S. Congenital toxoplasmosis. Neonatal Netw. 2001 Jun;20(4):23-30. [Internet]. [acesso em $2021 \mathrm{Fev}$ 1]. doi: 10.1891/0730-0832.20.4.23.

9. Jones JL, Dargelas V, Roberts J, Press C, Remington JS, Montoya JG. Risk for toxoplasma gondi Infection in the United States. Clin Infect Dis. 2009;49(6):878-84. [Internet]. [acesso em $2021 \mathrm{Fev}$ 1]. doi: $10.1086 / 605433$.

10. Antinarelli LMR, Silva MR, Guimarães RJP, Terror MS, Lima PE, Ishii JSC, et al. Rural residence remains a risk factor for toxoplasma infection among pregnant women in a highly urbanized brazilian area: a robust cross-sectional study. Trans R Soc Trop Med Hyg. 2021 Aug 2;115(8):896-903. [Internet]. [acesso em $2021 \mathrm{Fev}$ 1]. doi: 10.1093/trstmh/traa153.

11. Garnelo L, Horta BL, Escobar AL, Santos RV, Cardoso AM, Welch JR, et al. Avaliação da atenção pré-natal ofertada às mulheres indígenas no Brasil: achados do primeiro inquérito nacional de saúde e nutrição dos povos indígenas. Cad Saude Publica. 2019 Aug 19;35(3):eoo181318. [Internet]. [acesso em 2021 Fev 1]. doi: 10.1590/o102-311Xoo181318.

12. American Academy of Pediatrics. Report of the Committee on Infectious Diseases. In: Kimberlin DW, Brady MT, Jackson MA, Long SS (editores). Red Book. 31a ed. Itasca: American Academy of Pediatrics; 2018.

13. Prusa AR, Kasper DC, Pollak A, Gleiss A, Waldhoer T, Hayde M. The Austrian toxoplasmosis register, 1992-2008. Clin Infect Dis. 2015 Jan 15;60(2):e4-10. . [Internet]. [acesso em 2021 Fev 1]. doi: $10.1093 /$ cid/ciu724.

14. Nissen J, Jokelainen P, Rune Stensvold C, Trevisan C, Fuchs J, Sølvsten Burgdorf K, et al. The disease burden of congenital toxoplasmosis in Denmark, 2014. PLoS One. 2017;12(5):e0178282. . [Internet]. [acesso em $2021 \mathrm{Fev}$ 1]. doi: 10.1371/journal.pone.0178282. 
15. Brasil. Ministério da Saúde. Atenção à saúde do recém-nascido: guia para os profissionais de saúde. Vol. 2. Brasília: Ministério da Saúde; 2014. . [Internet]. [acesso em 2021 Fev 1]. Disponível em: https://bvsms.saude.gov.br/bvs/publicacoes/atencao_saude_recem_nascido_v2.pdf.

16. Brasil. Lei no $\mathbf{1 4 . 1 5 4}$, de 26 de maio de 2021. Altera a Lei no 8.069, de 13 de julho de 1990 (Estatuto da Criança e do Adolescente), para aperfeiçoar o Programa Nacional de Triagem Neonatal (PNTN), por meio do estabelecimento de rol mínimo de doenças a serem rastreadas pelo teste do pezinho; e dá outras providências. . [Internet]. [acesso em 2021 Fev 1]. Disponível em: https://www.in.gov.br/ en/web/dou/-/lei-n-14.154-de-26-de-maio-de-2021-322209993.

17. Santa Catarina. Secretaria de Estado da Saúde. Sistema Único de Saúde. Superintendência de Vigilância em Saúde. Diretoria de Vigilância Epidemiológica. Nota Técnica Conjunta nºo8/2019 DAPS/DIAF/DIVE/LACEN. . [Internet]. [acesso em $2021 \mathrm{Fev}$ 1]. Disponível em: https://dive.sc.gov. br/notas-tecnicas/docs/Nota_tecnica_toxoplasmose_versao_07_11-19.pdf. 
